\title{
Propagations of Sin-Gaussian Beam with Astigmatism through Oceanic Turbulence
}

\author{
Kaicheng Zhu ${ }^{1,3}$, Chang Gao ${ }^{1}$, Jiahui $\mathrm{Li}^{1}$, Dengjuan $\mathrm{Ren}^{1}$ and Jie $\mathrm{Zhu}^{2, *}$ \\ 1School of Engineering, Guangzhou College of Technology and Business, Guangzhou 510850, China \\ ${ }^{2}$ College of Science, Guizhou Institute of Technology, Guiyang 550003, China \\ ${ }^{3}$ School of Physics and Electronics, Central South University, Changsha 410083, China
}

\begin{abstract}
The propagation behaviours of a sin-Gaussian beam (SiGB) with astigmatism in oceanic water is analysed. The analytical expressions for the average intensity of such a beam are derived by using the extended Huygens-Fresnel integral. Its average intensity and on-axial intensity distributions in oceanic water are numerically examined. Then, we mainly focus on the effect of the beam parameters and the medium structure constant on the propagation behaviours for the astigmatic SiGBs in oceanic water, revealing that the evolutions of the intensity distributions can be effectively modulated by adjusting the astigmatic parameter, coherence length and the atmosphere turbulence strength.
\end{abstract}

\section{Introduction}

Aberrations can emerge when the laser light propagates through an imperfect optical system, or can be inherent like in laser diode. Many theoretical and experimental works have been conducted to investigate the propagation properties and beam quality of laser beams with astigmatic aberration through ABCD optical system or oceanic water [1-5]. For instance, it has been reported that designing spiral phase plate can give rise to the optical vortex beams [2-4], and a focused elliptical Gaussian beam carrying specified phase structure without circular symmetry can possess large amounts of orbital angular momentum [6]. Note that the phase shown in the initial sin-Gaussian beams can have great impact on the phase singularities and the pattern profiles during transmission, proposing an approach for improving the quality of phase singular beam through designing the phase configuration of illuming beam. Very recently, a few papers focus on how added phase of the illuming light influences the intensity and phase properties during propagation in various isotropic or anisotropic media [7]. It has been shown that, when a dark hollow beam has a small beam waist size, appropriate astigmatism can effectively decrease its scintillation index value particularly in the distant field [19].

In the past years, increasing attention has been drawn to various types of beam models in oceanic water owing to their significant applications in laser radar, optical communications, optical imaging, remote detection, and so on [1]. In [8,9], the application of partially coherent beam in laser communication has been analysed, revealing that prior to

*Corresponding author: jiezh_16@163.com 
the coherent models, the partially coherent beams can effectively control the bit error rate of the optical communication links. Therefore, there have been considerable research activities focusing on the partially coherent beams travelling through oceanic turbulence [10-18]. For instance, Hermite-sinusoidal-Gaussian beams are known to be derived from the paraxial wave equation, containing massive classes of beams like sinh-(or cosh-) Gaussian and sin-(or cos-) Gaussian types $[19,20]$ and such beams propagating through oceanic water or optical system have been extensively studied [10-18]. The correlation features of Hermite-sine-Gaussian and Hermite-sinh-Gaussian beams in oceanic water have been examined $[11,14]$. Theoretical models for describing the reciprocity properties of sineGaussian and sinh-Gaussian beams in oceanic water have been proposed [10]. The relationship of the oceanic system and the beam quality of sine-Gaussian lights have been investigated [12]. The spreading and the average intensity of the sinh-Gaussian beams in oceanic turbulence have been explored [12]. Besides, the scintillation index of sineGaussian and sinh-Gaussian beams has been investigated in great detail [13]. Very recently, Li et al. explore the dynamic evolution behaviour of the sine-Gaussian vortex beams and sine-Gaussian non-vortex beams travelling through free space and oceanic turbulence [17]. However, note that most of the aforesaid studies have ignored the aberration conditions.

In this work, we take the paraxial form of the extended Huygens-Fresnel principle for our study and analyse the propagation mechanism of SiGBs in oceanic water. The nonclassical propagation properties such as the intensity distribution and the spreading of the SiGBs are presented numerically by the analytically derived expressions. Some comparisons of the average intensity distribution between astigmatic or stigmatic SiGBs are made.

\section{Propagation of a partially coherent SiGB with astigmatism in atmospheric turbulence}

In the Cartesian coordinate system, the z-axis is designated as the propagation axis. According to [3], assume the SiGB with astigmatism at the source plane ( $\mathrm{z}=0)$ to be the following form

$$
E(x, y)=E_{0} \exp \left(-\frac{x^{2}+y^{2}}{w_{0}^{2}}\right) \sin \left(\frac{a x}{w_{0}}\right) \sin \left(\frac{b y}{w_{0}}\right)
$$

where $w_{0}$ is the waist width of the Gaussian part, $a$ and $b$ are the coefficients of the sinusoidal function. In the following, we concentrate on the astigmatism case where $a$ and $b$ are not equal. Besides, note that $E_{0}^{-2}=\pi w_{0}^{2}\left(1-e^{-a^{2} / 2}\right)\left(1-e^{-b^{2} / 2}\right) / 8$ makes the total power of the beam to be one unit.

The cross-spectral density of the SiGB travelling through oceanic water at propagation distance $\mathrm{z}$ can be calculated according to the formula based on the extended HuygensFresnel principle: 


$$
\begin{aligned}
W\left(x_{1}, y_{1}, x_{2}, y_{2}, z\right)= & \frac{1}{\lambda^{2} z^{2}} \int d x_{10} d y_{10} d x_{20} d y_{20} W\left(x_{10}, y_{10}, x_{20}, y_{20}, 0\right) \\
& \exp \left\{\frac{i k}{2 z}\left[\left(x_{1}-x_{10}\right)^{2}+\left(y_{1}-y_{10}\right)^{2}-\left(x_{2}-x_{20}\right)^{2}-\left(y_{2}-y_{20}\right)^{2}\right]\right\} \\
& \left\langle\exp \left[\psi^{*}\left(x_{10}, y_{10}, x_{1}, y_{1}\right)+\psi\left(x_{20}, y_{20}, x_{2}, y_{2}\right)\right]\right\rangle_{m}
\end{aligned}
$$

where $\mathrm{k}=2$ with being the wavelength, $\psi\left(x_{0}, y_{0}, x, y\right)$ is the solution for the Rytov method that indicates the random component of the complex phase for a spherical wave propagating from the source plane $(\mathrm{z}=0)$ to the measurement plane, and the angle bracket represents the ensemble average over the medium statistics including the phase fluctuations and the log-amplitude due to the oceanic turbulence In this paper, Kolmogorov spectrum and a quadratic approximation of the 5/3 power law for Rytov's phase structure function are employed. The last term in the integrand of Eq. (2) can be written as

$$
\left\langle\exp \left[\psi^{*}\left(x_{1}, y_{1}, x, y\right)+\psi\left(x_{2}, y_{2}, x, y\right)\right]\right\rangle_{m}=\exp \left[-\frac{\left(x_{1}-x_{2}\right)^{2}+\left(y_{1}-y_{2}\right)^{2}}{\rho_{0}^{2}}\right]
$$

with $\rho_{0}$ being the spatial coherence radius for a sphere wave transmitting through the oceanic water written as

$$
\begin{aligned}
\rho_{0}^{-2} & =\frac{\pi^{2} k^{2} z}{3} \int_{0}^{\infty} \kappa^{3} \Phi_{n}(\kappa) d \kappa \\
& =1.801 C_{n}^{2} k^{2} z \eta^{-1 / 3}\left(0.483-0.835 \varpi^{-1}+3.85 \varpi^{-2}\right)
\end{aligned}
$$

where $\Phi_{n}(\kappa)$ is the spatial power spectrum of the oceanic water, which can be expressed by the "equivalent" temperature structure constant $C_{n}^{2}$ related to $\varpi$ with a unit $m^{-2 / 3} K^{2} \mathrm{~m}-2 / 3 \mathrm{~K} 2$.

We point out that in this paper only the average intensity of the astigmatic SiGB at the receiver plane after propagating distance $\mathrm{z}$ in the oceanic turbulence is considered. Then, we can take $\mathrm{x} 1=\mathrm{x} 2$ and $\mathrm{y} 1=\mathrm{y} 2$, directly reducing the cross-spectral density $W\left(x_{1}, y_{1}, x_{2}, y_{2}, z\right)$ for a partially coherent $\operatorname{SiGB}$ to its average intensity $I(x, y, z)$. Substituting Eqs.(2)-(4) into Eq. (3) and introducing the following "sum" and "difference" coordinates

$$
q_{s 0}=\frac{q_{10}+q_{20}}{2 w_{0}} \quad(q=x, y) q_{d 0}=\frac{q_{10}-q_{20}}{w_{0}} \quad(q=x, y)
$$

we obtain 


$$
\begin{aligned}
I(x, y, z) & =\frac{E_{0}^{2} w_{0}^{4}}{2 \lambda^{2} z^{2}} \int d x_{s 0} d y_{s 0} d x_{d 0} d y_{d 0}\left[\cos \left(a x_{d 0}\right)-\cos \left(2 a x_{s 0}\right)\right]\left[\cos \left(a y_{d 0}\right)-\cos \left(2 a y_{s 0}\right)\right] \\
& \exp \left[-2 x_{s 0}^{2}-2 y_{s 0}^{2}-\Omega\left(x_{d 0}^{2}+y_{d 0}^{2}\right)+2 i Z\left(x_{s 0} x_{d 0}+y_{s} y_{d 0}-x_{w} x_{d 0}-y_{w} y_{d 0}\right)\right] \\
= & \frac{E_{0}^{2} Z^{2}}{2 \pi^{2}}\left(I_{d x}-I_{s x}\right)\left(I_{d y}-I_{s y}\right) \\
& \Omega=\frac{w_{0}^{2}}{\rho_{0}^{2}}+\frac{w_{0}^{2}}{2 \delta_{0}^{2}}+\frac{1}{2}, Z=\frac{z_{R}}{z}, z_{R}=\frac{k w_{0}^{2}}{2}=\frac{\pi w_{0}^{2}}{\lambda} \quad \text { is the Rayleigh distance }
\end{aligned}
$$
and $\mathrm{qw}=\mathrm{q} / \mathrm{w} 0(\mathrm{q}=\mathrm{x}, \mathrm{y})$ are the scaled coordinates on the receiver plane. Further, note the following integral formula

$$
\int_{-\infty}^{\infty} \exp \left(-\alpha x^{2}+\beta x\right) d x=\sqrt{\frac{\pi}{\alpha}} \exp \left(\frac{\beta^{2}}{4 \alpha}\right)
$$

we finally obtain

$$
\begin{aligned}
& I_{d x}=\frac{\pi}{\sqrt{2 \Omega+Z^{2}}} \exp \left(-\frac{2 Z^{2} x_{w}^{2}+a^{2} / 2}{2 \Omega+Z^{2}}\right) \cosh \left(\frac{a Z x_{w}}{2 \Omega+Z^{2}}\right) \\
& I_{d y}=\frac{\pi}{\sqrt{2 \Omega+Z^{2}}} \exp \left(-\frac{2 Z^{2} y_{w}^{2}+b^{2} / 2}{2 \Omega+Z^{2}}\right) \cosh \left(\frac{b Z y_{w}}{2 \Omega+Z^{2}}\right) \\
& I_{ \pm s x}=\frac{\pi}{\sqrt{2 \Omega+Z^{2}}} \exp \left(-\frac{2 Z^{2} x_{w}^{2}+\Omega a^{2}}{2 \Omega+Z^{2}}\right) \cos \left(\frac{2 a Z^{2} x_{w}}{2 \Omega+Z^{2}}\right) \\
& I_{s y}=\frac{\pi}{\sqrt{2 \Omega+Z^{2}}} \exp \left(-\frac{2 Z^{2} y_{w}^{2}+\Omega b^{2}}{2 \Omega+Z^{2}}\right) \cos \left(\frac{2 b Z^{2} y_{w}}{2 \Omega+Z^{2}}\right)
\end{aligned}
$$

Equations (6), (8)-(11) construct the main analytical result of our research, which will provide an accurate and simple method for exploring the propagation characteristics of a $\mathrm{SiGB}$ with or without astigmatism (separately featured by $\mathrm{a}=\mathrm{b}$ or not) in free space or in the oceanic water (respectively corresponding to $C_{n}^{2}=0$ or not).

\section{Numerical results and analysis}

In this section, we mainly study the evolution of the average intensity of SiGB with astigmatism transmitting in oceanic water numerically and comparatively by using the analytical result derived in the previous calculation. Without loss of generality, the wavelength and the beam width are set to be: $\quad 800 \mathrm{~nm}$ and $\mathrm{w} 0=50 \mathrm{~mm}$, respectively, in the following discussions, and the corresponding Rayleigh distance $\mathrm{zR} \approx 9.82 \mathrm{~km}$. 

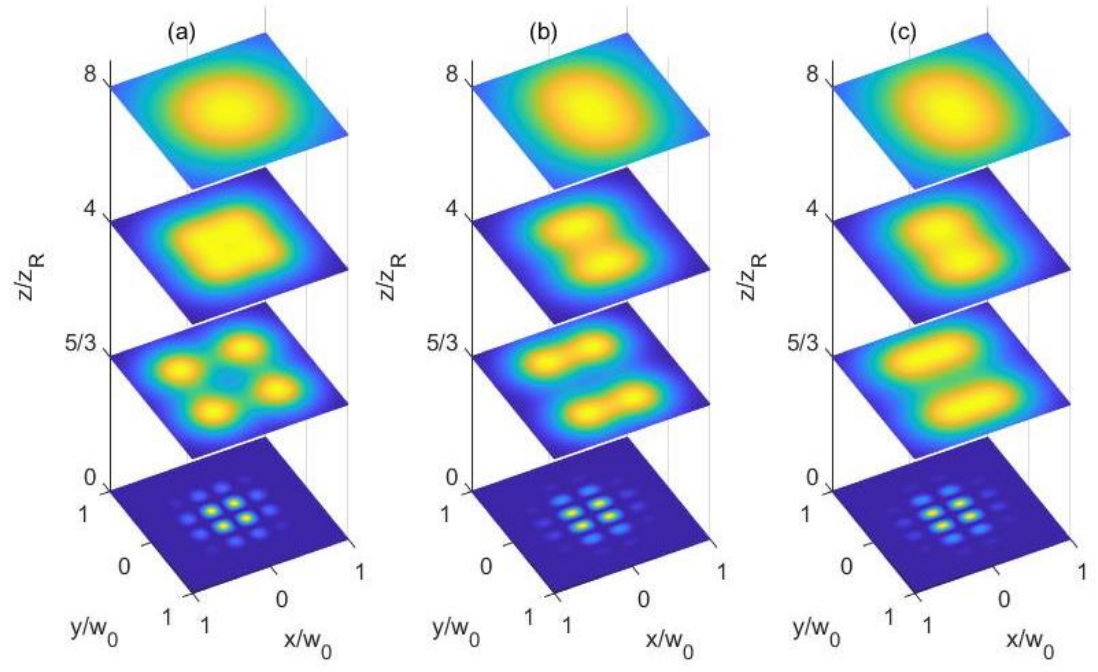

Fig.1. On the different receiving planes average intensity distributions of SiGBs without or with astigmatism propagating through oceanic water, where $C_{n}^{2}=8 \times 10^{-16} \mathrm{~m}^{-2 / 3}$ (a) $a=b=5, d_{0}=\infty$; (b) $a=4, b=6, d_{0}=\infty$ and (c) $a=4, b=6, \delta_{0}=w_{0} / 5$.

Figure 1(a) shows the numerical calculation results of the intensity distribution evolutions for initial coherent $\operatorname{SiGB}(\mathrm{d} 0=\infty)$ without stigmatism $(\mathrm{a}=\mathrm{b}=2)$ propagating in oceanic water. The intensity distribution $\mathrm{I}(\mathrm{x}, \mathrm{y}, \mathrm{z})$ is a four-petal profile in the near field and gradually evolves into a Gaussian-like configuration after travelling a sufficiently long distance. Figures 1 (b) and (c) illustrate the intensity distribution $\mathrm{I}(\mathrm{x}, \mathrm{y}, \mathrm{z})$ of a SiGB with stigmatism for $a=1$ and $b=3$ with different values of $d 0$. We point out that Fig.1(b) corresponds to the initial coherent beam $(\mathrm{d} 0=\infty)$ but Fig.1(c) to the initial beam. Obviously, the intensity pattern of a SiGB with astigmatism gradually fuses into a two-petal configuration in the near field and finally evolves into Gaussian-like intensity configuration after travelling a sufficiently long distance. In fact, performing calculations indicate that, so long as the astigmatism exist in which $a \neq b$, the intensity profile of an astigmatic SiGB always exhibits the similar evolution behaviour aforesaid during the propagation in oceanic water. By way of comparison Figs. 1(b) with 1(c) it is also demonstrated that the intensity evolutions of the SiGB propagating in oceanic water show great difference.

We further investigate the variation of on-axis intensity of such SiGBs with propagating distance in the oceanic water. As well known, the on-axial intensity of a SiGBs with or without astigmatism is always zero during the propagation in free space. Figure 2 shows the dependences of the on-axial intensity of SiGBs with or without astigmatism on the propagation distance $z$. Obviously, the on-axial intensity grows rapidly from zero with the increase of propagating distance, and reaches a maximum at a specified distance zs. When the propagating distance is further increased, the on-axial intensity smoothly decreases and eventually approaches to 0 at a sufficiently long distance. Additionally, a comparison of Figs. 1(a) and 1(b) confirms that the value of the maximum on-axial intensity and the position of such maximum value depend on the astigmatic parameter, the turbulence strength parameter $C_{n}^{2}$ as well as the coherence length $\mathrm{d} 0$. 

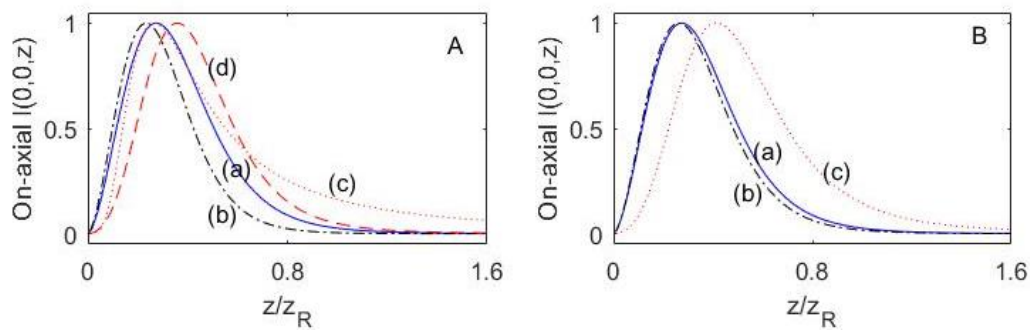

Fig.2. On-axial intensity of SiGBs without or with astigmatism propagating through oceanic water, where $C_{n}^{2}=1 \times 10^{-16} \mathrm{~m}^{-2 / 3}$ and A: (a) $a=b=3, d_{0}=\infty$; (b) $a=1, b=5, d_{0}=\infty$; (c) $a=1, b=5$, $\delta_{0}=w_{0} / 5$ and (d) $a=1, b=5, \delta_{0}=10 w_{0}$; B: (a) $a=b=3, d_{0}=\infty$; (b) $a=2, b=4, d_{0}=\infty$; (c) $a=2, b=4$, $\delta_{0}=10 w_{0}$.

\section{Conclusions}

In summary, we have formulated the effects of the astigmatism on the average intensity distribution of SiGBs propagating in the oceanic water. The analytical expressions of the average intensity are derived. According to the numerical calculations, we find that, under the suitable parameter conditions (astigmatic parameter, the turbulence strength parameter $C_{n}^{2}$ ), the average intensity pattern of an astigmatic SiGB experiences many variations including two-petal, flopped and Gaussian-like configurations during propagation. It is proved that the structure constant $C_{n}^{2}$ and the astigmatic parameter can greatly influence the variation of intensity patterns. Therefore, the results drawn in the paper may have remarkable potential applications such as laser communication in free space or oceanic water and laser illumination.

\section{References}

[1] Korotkova O 2014 Random Light Beams Theory and Applications (CRC Press)

[2] Kotlyar V V, Kovalev A A and Porfirev A P 2019 Vortex Laser Beams (CRC Press)

[3] Tang H Q and Zhu K C 2013 Opt. \& Laser Technology 3 68-71

[4] Zhu K C, Tang H Q, Tang Y and Xia H 2014 Opt. \& Laser Technol. 64 11-16

[5] Shen S, Yang Z J, Guo J L, Wang Y J and Pang Z G 2020 Optik 224165454

[6] Courtial J, Dholakia K, Allen L and Padget M J 1997 Opt. Commun. 144 210-213

[7] Kotlyar V V, Kovalev A A, Porfirev A P 2018 Opt. Express 26(1) 141-156

[8] Ricklin J C and Davidson F M 2002 J. Opt. Soc. Am. A 19(9) 1794-1802

[9] Ricklin J C and Davidson F M 2003 J. Opt. Soc. Am. A 20(5) 856-866

[10] Eyyuboglu H T and Baykal Y 2005 J. Opt. Soc. Am. A 22 2709-2718

[11] Baykal Y J 2006 J. Opt. Soc. Am. A 23 889-893

[12] Xiao X, Ji X and Lü B 2008 Opt. Laser Technol. 40 129-136

[13] Cai Y, Eyyuboğlu H T and Baykal Y J 2008 J. Opt. Soc. Am. A 25 1497-1503

[14] Baykal Y 2012 J. Opt. 14075707 
[15] Saad F, Halba E. M. E and Belafhal A 2017 Opt. Quant. Electron. 4994

[16] Pan Y T, Wang P, Wang W, Li S, Cheng M J and Guo L X 2021 Opt. Express 29 $12644-12662$

[17] Li J H, Zeng J, Duan M L 2015 Opt. Express 23 11556-11565

[18] Zhu J, Zhu K C, Tang H Q and Xia H 2017 J. Mod. Opt. 64 1915-1921

[19] Casperson L W, Hall D G and Tovar A A 1997 J. Opt. Soc. Am. A 14 3341-3348

[20] Casperson L W and Tovar A A 1998 J. Opt. Soc. Am. A 15 954-961 